ESAIM: PROCEEDINGS AND SURVEYS, September 2014, Vol. 45, p. 108-117

J.-S. Dhersin, Editor

\title{
A NEW MODEL FOR SHALLOW VISCOELASTIC FREE-SURFACE FLOWS FORCED BY GRAVITY ON ROUGH INCLINED BOTTOM
}

\author{
SÉBASTIEN BOYAVAL ${ }^{1}$
}

\begin{abstract}
A thin-layer model for shallow viscoelastic free-surface gravity flows on slippery topographies around a flat plane has been derived recently in [Bouchut-Boyaval, M3AS (23) 2013]. We show here how the model can be modified for flows on rugous topographies varying around an inclined plane.

The new reduced model extends the scope of one derived in [Bouchut-Boyaval, M3AS (23) 2013]. It is one particular thin-layer model for free-surface gravity flows among many ones that can be formally derived with a generic unifying procedure. Many rheologies and various shallow flow regimes have already been treated within a single unified framework in [Bouchut-Boyaval, HAL-ENPC (00833468) 2013]. The initial full model used here as a starting point is however a little different to one used in [Bouchut-Boyaval, HAL-ENPC (00833468) 2013], although the new thin-layer model is very similar to the one derived therein. Precisely, here, the bulk dissipation (due to e.g. viscosity) is neglected from the beginning, like in [Bouchut-Boyaval, M3AS (23) 2013].

Moreover, unlike in [Bouchut-Boyaval, HAL-ENPC (00833468) 2013], we perform here numerical simulations. The interest of the extension is illustrated in a physically interesting situation where new stationary solutions exist. To that aim, the Finite-Volume method proposed in [Bouchut-Boyaval, M3AS (23) 2013] needs to be modified, with an adequate discretization of the new source terms. Interestingly, we can also numerically exhibit an apparently new kind of "roll-wave" solution.
\end{abstract}

\section{INTRODUCTION}

Given a relaxation-time $\lambda>0$ and an elastic stress relaxation modulus $G>0$ as physical parameters for the rheology of a viscoelastic fluid, two-dimensional (2D) viscoelastic flows can be modelled by the incompressible Euler/Upper-Convected-Maxwell (UCM) equations

$$
\left\{\begin{array}{l}
\left(\partial_{t}+\boldsymbol{u} \cdot \boldsymbol{\nabla}\right) \boldsymbol{u}+\boldsymbol{\nabla} p=G \operatorname{div}(\boldsymbol{\sigma})+\boldsymbol{f}, \\
\operatorname{div} \boldsymbol{u}=0, \\
\lambda\left(\left(\partial_{t}+\boldsymbol{u} \cdot \boldsymbol{\nabla}\right) \boldsymbol{\sigma}-(\boldsymbol{\nabla} \boldsymbol{u}) \boldsymbol{\sigma}-\boldsymbol{\sigma}(\boldsymbol{\nabla} \boldsymbol{u})^{T}\right)=\boldsymbol{I}-\boldsymbol{\sigma} .
\end{array}\right.
$$

In the case of free-surface gravity flows at a regional scale, the system (1.1) is considered in a time-dependent domain $\mathcal{D}(t)$ of $\mathbb{R}^{2}$ endowed with a fixed Cartesian frame of coordinates $\left(\boldsymbol{e}_{x}, \boldsymbol{e}_{z}\right)$ such that $\boldsymbol{f}=g(\sin \theta,-\cos \theta)$ is a uniform gravity force field with $\theta \in[0, \pi / 2)$ constant. We have denoted by $\boldsymbol{u}=(u, w)$ the velocity, $p$ the pressure, and $\boldsymbol{\sigma}$ the conformation tensor of the viscoelastic material with internal stress (I being the 2D identity matrix)

$$
\boldsymbol{\tau}=G(\boldsymbol{\sigma}-\boldsymbol{I})
$$

1 Université Paris-Est, Laboratoire d'hydraulique Saint-Venant, Ecole Nationale des Ponts et Chaussées - EDF R\&D CETMEF, 6 quai Watier, 78401 Chatou Cedex, France \& MATHERIALS, INRIA Rocquencourt (sebastien.boyaval@enpc.fr)

(C) EDP Sciences, SMAI 2014 
Here, we consider shallow flows, for which the $2 \mathrm{D}$ model above can be simplified into a $1 \mathrm{D}$ reduced model with less degrees of freedom. The simplification procedure for shallow flows is formal, since rigorous error bounds between the full and reduced models remain difficult. Though, in [4], one has nevertheless been able to simplify (1.1) into a 1D reduced model within a sound mathematical framework, following the lines proposed in [8] for the model reduction of shallow Newtonian viscous flows. In particular, sufficient conditions can be precisely formulated to consistently (i) neglect the vertical velocity variable $w \equiv \boldsymbol{u} \cdot \boldsymbol{e}_{z}$ in (1.1), and (ii) average out the vertical variations of the variables $u \equiv \boldsymbol{u} \cdot \boldsymbol{e}_{x}$ and $\boldsymbol{\sigma}$ along $\boldsymbol{e}_{z}$, after supplementing (1.1) with Boundary Conditions (BCs) for free-surface flows on slippery topographies, when $\theta=0$. It was also noted in [4] that the choice of BCs is crucial in the formal simplification procedure.

Precisely, to deal with shallow free-surface flows, one considers a layer geometry comprised in between a kinematic free-surface $(t, x) \mapsto b(x)+h(t, x)$ and an impermeable topography $x \mapsto z=b(x)$ for all $x \in$ $(-L / 2, L / 2)$ and $t \in(0, T)$, given $T>0$ and $L>0$, that is

$$
\mathcal{D}(t)=\{(x, z), \quad x \in(-L / 2, L / 2), \quad 0<z-b(x)<h(t, x)\}
$$

Then, sufficient conditions for the simplification of the equations (1.1) in $\mathcal{D}(t)$ are formulated through an asymptotic regime. In [4], a 1D reduced model was obtained as the limit of a family of fluid flows such that, in a fixed space-time range with characteristic sizes $L \times T$, when $\theta=0$ :

- the elastic stress relaxation modulus $G$ is infinitely small compared with $(L / T)^{2}$,

- the layer depth is equally infinitely small $h \ll L$,

- and the variations of the bottom topography $\partial_{x} b$ are also infinitely small.

Moreover, in [4], a no-tension condition was required at the free-surface $z=b(x)+h(t, x)$, as well as a pure-slip condition at the bottom $z=b(x)$. The result is a $1 \mathrm{D}$ elongational model for free-surface viscoelastic flows. Questions then naturally arise. Can we change the BCs? Can we get a 1D reduced model in more or different flow regimes than elongational ? As a matter of fact, numerous variations of [4] have already been treated in [5].

It is shown in [5] that one can use a formal consistency argument with clear mathematical assumptions as a generic procedure to univoquely define various reduced fluid models in a single unified framework. Other BCs than pure-slip, and possibly sheared (as opposed to purely elongational) free-surface gravity flows, have already been considered in [5] for viscoelastic fluids, in a number of various shallow regimes. In particular, a reduced model for viscoelastic fluids like in [4] has been derived in [5] on requiring a Navier friction BC

$$
\boldsymbol{\tau} \boldsymbol{n}-(\boldsymbol{\tau} \boldsymbol{n} \cdot \boldsymbol{n}) \boldsymbol{n}=k(\boldsymbol{u}-(\boldsymbol{u} \cdot \boldsymbol{n}) \boldsymbol{n}) \quad \text { at } z=b(x)
$$

where $\boldsymbol{n}$ denotes the unit normal toward the flow and $k>0$ the friction coefficient. Note that the condition (1.2) is more general than pure-slip: one recovers the pure-slip case when $k \rightarrow 0$, but covers other physically meaningful cases with rugous topographies when $k>0$ otherwise. Though, the 3D Navier-Stokes/UCM equations were used in [5] as a starting point ${ }^{1}$, instead of the 2D Euler/UCM equations (1.1) here like in [4].

In Section 2, we derive a thin-layer 1D reduced model using (1.2) instead of pure-slip $(k=0)$, and starting with the 2D Euler/UCM equations (1.1) like in [4]. The 1D reduced model obtained here can be compared to a $2 \mathrm{D}$ reduced model obtained in [5]. But one recovers it exactly only after assuming an additional translation invariance, and letting the additional (second-order) viscous dissipative terms vanish, in the 2D reduced model of [5]. The procedure is much simpler here, and still useful in the cases where bulk dissipation can be neglected. One does not even have to precise explicitly the form of the bulk dissipation term in the initial model here. However, we have to require a stronger assumption $k \ll L / T$ than in [5]. So shear effects are still second-order in the present 1D reduced model here, like in [4] where $k=0$. Yet, the new $1 \mathrm{D}$ reduced model when $k>0$ is not a useless extension. First, $k \ll L / T$ is only a sufficient (not necessary) condition in the formal derivation of the $1 \mathrm{D}$ reduced model. Second, the new friction term at bottom is a sufficient dissipative phenomenon to

\footnotetext{
${ }^{1}$ In terms of viscoelastic rheology, adding viscous dissipation in (1.1) also translates as a non-zero retardation time in the UCM equations, see [5].
} 
balance gravity forcing in the $1 \mathrm{D}$ reduced model. The new reduced model thus has new stationary solutions when $k>0$ on inclined topographies where $\theta \in\left(0, \frac{\pi}{2}\right)$. (In [4], only the case $\theta=0$ was investigated.)

In Section 3, we show numerical simulations of the new 1D reduced model in interesting physical situations. (Notice that no numerical simulations were performed in [5] for any variation of the 1D reduced model of [4].) To discretize the new source terms such that friction balances the gravity forcing, and the new stationary states can be captured numerically, we propose a modification of the Finite Volume (FV) scheme that was constructed in [4] to simulate slippery topographies $(k=0)$ when $\theta=0$.

Remark 1. Our simplification procedure is inspired by that in [8] for Newtonian viscous flows modelled by the Navier-Stokes equations. In [8], the well-posedness of the initial full system of equations is then clearly a reason why one requires conditions for all components of the stress tensor at the boundary. The situation is similar in [5], where the initial full system of equations always includes some viscous dissipation before simplification. Now, here and in [4], the initial full model is Euler system of equations. Dynamic conditions then seem unnecessary in the direction tangent to the boundary. In particular, the pure-slip BC in [4], or the friction $B C$ (1.2) here, seem additional constraints to the solutions of an a priori well-posed BVP. And one additional difference in between the models derived here or in [4], and in [5], may be the status of BCs. Though, this is not so clear as long as no complete well-posedness theory exists. For instance, dissipative boundary conditions could allow one to discriminate between multiple discontinuous solutions in Euler case.

\section{Derivation of the MOdeL}

We follow the procedure proposed in [4] to simplify the model (1.1) into a new one. The new model shall be simpler in the sense that it uses less unknown variables, and the remaining unknown variables shall moreover depend on less coordinates.

The procedure consists in first defining formally a family of solutions to the initial model (1.1) that is indexed by an adimensional parameter $\varepsilon>0$, such that the terms appearing in the system of equations are functions scaled by $\varepsilon \rightarrow 0$ everywhere in space and time (that is uniformly, given a fixed time-space range).

To define such a family of solutions indexed by $\varepsilon \rightarrow 0$, the data is first scaled by $\varepsilon$. For instance we assume $G=O(\varepsilon)$ here, the equivalent assumption to $\eta_{p}:=2 G \lambda=O(\varepsilon)$ in [4]. When the initial model (1.1) is clearly well-posed, one may hope that this is enough. Though, it seems that thin-layer fluid models also require additional regularity assumptions on the solution itself, which cannot yet be directly connected to the data necessary for well-posedness. To proceed, as usual, we thus identify a shallow "flow regime" where the initial model (1.1) can be simplified into a reduced model by neglecting terms in a coherent way (see [5]).

(1) In thin-layer geometries where $h=O(\varepsilon)$, the momentum equation in (1.1) projected along $\boldsymbol{e}_{z}$ reads

$$
\partial_{z} p=G \partial_{z} \sigma_{z z}-g \cos \theta
$$

after neglecting $O(\varepsilon)$ terms. The fluid incompressibility and the impermeability of the smoothly varying bottom indeed imply $w=O(\varepsilon)$ together. With the no-tension $\mathrm{BC}$ at free-surface $z=b+h$, an approximation of $p=O(\varepsilon)$ then reads

$$
p(t, x, z)=G \sigma_{z z}(t, x, z)-g \cos \theta(b(x)+h(t, x)-z) .
$$

(2) Assuming $\partial_{z} u=O(1)$, the momentum equation projected along $\boldsymbol{e}_{x}$ reads, on noting $\sigma_{x z}=O(\varepsilon)$ because of BCs at $z=b$ and $z=b+h$ when $k=O\left(\varepsilon^{2}\right)$, and denoting $\tilde{g}=g \cos \theta$,

$$
\left(\partial_{t}+u \partial_{x}\right) u-\tilde{g} \tan \theta=O(\varepsilon) .
$$

Moreover, $\sigma_{x z}=O(\varepsilon)$ implies a stronger motion-by-slice $\partial_{z} u=O(\varepsilon)$ than assumed, and depthaverageing (2.1) over $z \in(b, b+h)$ yields, using no-tension at $z=b$ and Navier-slip at $z=b+h$ :

$$
h\left(\partial_{t}+u \partial_{x}\right) u-h \tilde{g} \tan \theta=-h \tilde{g} \partial_{x}(b+h)+G \partial_{x}\left(h\left(\sigma_{x x}-\sigma_{z z}\right)\right)-k u,
$$


after neglecting $O\left(\varepsilon^{3}\right)$ terms, and assuming $\partial_{z} \boldsymbol{\sigma}=O(1)$.

Note that at this stage, the flow regime specified along the simplification procedure requires exactly the same scaling as in [4]. So we have not much improved here on the fact that the simplification (and thereby the 1D reduced model below) a priori applies to extensional flows, although a new friction term in (2.2) accounts for shear at the bottom, in addition to the bulk elongational stress. This is different to [5] where a less strong assumption $k=O(\varepsilon)$ suffices for simplification ${ }^{2}$, and one does not require a stronger motion-by-slice than $\partial_{z} u=O(1)$ nor a smaller shear than $\sigma_{x z}=O(1)$. Yet, we have nevertheless obtained here a simplification that enlarges a bit the scope of application of [4]. In particular, we obtain a new reduced model that may of course still hold in regimes beyond the one required above for consistency.

Using the fluid incompressibility (i.e. mass conservation), the kinematic BC at the free-surface $z=b+h$, and simplifications of the UCM equations consistent with the flow regime specified above, one indeed gets the closed system (2.3), which defines approximate solutions to (1.1) at the same order in $\varepsilon$ as in [4]:

$$
\left\{\begin{array}{l}
\partial_{t} h+\partial_{x}(h u)=0 \\
\partial_{t}(h u)+\partial_{x}\left(h u^{2}+\tilde{g} \frac{h^{2}}{2}+G h\left(\sigma_{z z}-\sigma_{x x}\right)\right)=-\tilde{g} h\left(\partial_{x} b-\tan \theta\right)-k u \\
\partial_{t} \sigma_{x x}+u \partial_{x} \sigma_{x x}-2 \sigma_{x x} \partial_{x} u=\left(1-\sigma_{x x}\right) / \lambda \\
\partial_{t} \sigma_{z z}+u \partial_{x} \sigma_{z z}+2 \sigma_{z z} \partial_{x} u=\left(1-\sigma_{z z}\right) / \lambda
\end{array}\right.
$$

The system (2.3) is very similar to (4.8) in [4]: it is its counterpart in a rotated framework (hence the modified "vertical" gravity acceleration coefficient $g \rightarrow \tilde{g}$ and the additional momentum source term $g h \sin \theta)$ with an additional source term $-k u$ to model friction with a coefficient $k>0$ at a rugous bottom boundary. Like in the case $\theta=0=k$ treated in [4], it holds for (2.3) that:

- the limit $G \rightarrow 0$ yields the inviscid Saint-Venant (or shallow-water) equations and passive tracers $\sigma_{x x / z z}$,

- the "singular" limit $\lambda \rightarrow 0$ (a "small Deborah number" limit in the viscoelastic literature) yields different systems depending on which variables are assumed bounded in the limit.

For instance, assuming $\tau_{x x}-2 \lambda \partial_{x} u$ and $\tau_{z z}-2 \lambda \partial_{x} u$ remain bounded when $\lambda \rightarrow 0$, the system (2.3) becomes two-dimensional with $G h\left(\sigma_{z z}-\sigma_{x x}\right) \rightarrow-h \eta_{p} \partial_{x} u$ in the momentum equation, which is exactly the viscous Saint-Venant (or shallow-water) system of equations with viscosity $\eta_{p}=4 G \lambda=O(\varepsilon)$. Of course, in both limit cases, the limit system is rotated by an angle $\theta$ and has an additional friction term compared to [4].

In comparison with [4], the new reduced model (2.3) has however one very interesting new feature. There exist new stationary solutions, in particular uniform steady states $u=U \in \mathbb{R}, h=H \in \mathbb{R}, \sigma_{x x}=1=\sigma_{z z}$ where $g H \tan \theta=k U$ on inclined planes $\left(\partial_{x} b=0\right)$ which are not necessarily at rest unlike when $\theta=0$. This is very important to many geophysical situations, in hydraulics for instance. Besides, having in mind geophysical applications to e.g. mudflows, we shall use next, for the numerical applications, Manning formula $k=g M^{2}|u| / h^{\frac{1}{3}}$ with a coefficient $M$ (like in hydraulics where the coefficient $k$ in the Saint-Venant equations is believed to account for turbulent dissipation at the boundaries, hence a specific scaling as a function of $u$ and $h$ ). Let us now explore numerically the reduced system (2.3) in a few relevant test cases, with an adequate discretization.

\section{Numerical Simulations}

Let us numerically approximate solutions to the system of partial differential equations (2.3) on a time-space domain $(t, x) \in[0, T] \times[-L / 2, L / 2]$ given a function $[-L / 2, L / 2] \ni x \mapsto b(x) \in \mathbb{R}$, parameters $\tilde{g} \geq 0, G \geq 0, \lambda>$ $0, \theta \in[0, \pi / 2)$ and an initial condition $\left\{h, u, \sigma_{x x}, \sigma_{z z}\right\}(t=0, x)=\left\{h^{0}, u^{0}, \sigma_{x x}^{0}, \sigma_{z z}^{0}\right\}(x)$ for $x \in[-L / 2, L / 2]$.

\footnotetext{
${ }^{2} \mathrm{~A}$ second-order approximation of $u$ can be defined consistently in [5] without assuming the boundary shear second-order too, because one can construct explicitly a first-order correction with the help of the explicit (viscous) bulk dissipation term.
} 
The quasilinear formulation of (2.3) is very similar to that of the reduced model in [4]. In fact, the homogeneous part of (2.3) - obtained when $\theta=0=k$ in the limit $\lambda \rightarrow+\infty$ with bounded variables $\sigma$-is exactly the same as in [4] (i.e. only the source terms in (2.3) are different). Then, we use here the same time-splitting approach as in [4] with the same first step to treat the homogeneous part of (2.3) (sources are treated next).

We recall that the system of equations is hyperbolic as long as solutions remain in the physical domain $\mathcal{U}=\left\{h \geq 0, \sigma_{x x}>0, \sigma_{z z}>0\right\}$. Moreover, smooth solutions to (2.3) remain in $\mathcal{U}$ when initialized in $\mathcal{U}$ at $t=0$ and this is necessary for the natural energy of the system to be well-defined, the so-called free energy

$$
E=h \frac{u^{2}}{2}+g \frac{h^{2}}{2}+\frac{G}{2} h\left(\sigma_{x x}+\sigma_{z z}-\ln \left(\sigma_{x x} \sigma_{z z}\right)-2\right) .
$$

Then $\mathcal{U}$ defines an invariant domain for (2.3) that we shall strive to maintain at the discrete level. Furthermore, although we note that (2.3) rewrites as a system of conservation laws after a change of variable, we follow [4] and use a Godunov-type Finite-Volume (FV) discretization of (2.3) - a way to tackle nonlinearities - for the system written in the non-conservative variable $q=\left(h, h u, h \sigma_{x x}, h \sigma_{z z}\right)$. Indeed, $E(q)$ is convex on the convex set $\mathcal{U}$, while this is not true when the system in written in a conservative form with other ("conservative") variables (see a proof in the appendix of [4]). On the one hand, choosing $q$ as the variable to discretize still naturally conserves the liquid mass $h$ discretely. Note that the evolution of $h$ is actually the single conservation law that is actually required by the physical interpretation of the reduced model. On the other hand, the convexity of the natural energy $E$ of the system, and the simple 3-wave structure of the homogeneous part of (2.3), which does have a conservative formulation, allow one to build a simple FV scheme for the latter that is both consistent and "stable" (in the sense of dissipative for the natural energy E). More precisely, we use Suliciu Riemann solver of relaxation type, whose dissipation properties can be analyzed in "Lagrange variables" (see [4] and $[2,3]$ ).

Defining FV approximate cell values for $q$ at discrete times $0<t^{1}<\ldots<t^{n}<t^{n+1}<\ldots<t^{N}=T$ as

$$
q_{i}^{n} \approx \frac{1}{\Delta x_{i}} \int_{x_{i-1 / 2}}^{x_{i+1 / 2}} q\left(t^{n}, x\right) d x, i \in \mathbb{Z}
$$

on a fixed grid of $\mathbb{R}$ with cells $\left(x_{i-1 / 2}, x_{i+1 / 2}\right)_{i \in \mathbb{Z}}$ of volumes $\Delta x_{i}=x_{i+1 / 2}-x_{i-1 / 2}$ and centers $x_{i}=\frac{x_{i-1 / 2}+x_{i+1 / 2}}{2}$, the intermediate cell values are computed in the first stage of our splitting approach of (2.3) through

$$
q_{i}^{n+1,-}=q_{i}^{n}-\frac{\Delta t^{n}}{\Delta x_{i}}\left(\mathcal{F}_{l}\left(q_{i}^{n}, q_{i+1}^{n}\right)-\mathcal{F}_{r}\left(q_{i-1}^{n}, q_{i}^{n}\right)\right)
$$

as solutions of Riemann problems for the homogeneous part of (2.3) (i.e. without source) on $\left[t^{n}, t^{n+1}\right.$ ), given the data $q_{i}^{n}$ at $t^{n}$. We use the same numerical fluxes $\mathcal{F}_{l}, \mathcal{F}_{r}$ as in [4], under the same $\frac{1}{2}$-CFL condition on $\Delta t^{n}=t^{n+1}-t^{n}$ (we refer to [4] for the exact formulas). We recall that the numerical fluxes $\mathcal{F}_{l}, \mathcal{F}_{r}$ defined in [4] do not conserve the conservative variables $\left(\sigma_{x x}^{-1 / 2} / h, \sigma_{z z}^{1 / 2} / h\right)$. But one can ensure that the scheme (3.2) numerically dissipates pointwise a discrete equivalent of the physical energy (3.1) of the system. It holds

$$
E\left(q_{i}^{n+1,-}\right) \leq E\left(q_{i}^{n}\right) \forall i \in \mathbb{Z}, n=0 \ldots N-1,
$$

as well as the conservation of the invariant domain $\mathcal{U}$, which is a natural discrete stability requirement with a view to preventing e.g. the numerical propagation of non-physical shocks by the FV approximation of $q$.

Next, in the second stage of the splitting approach, we treat the source terms, which are different from [4] and need a specific treatment. To that aim, recall that additionally to the problem of discretizing the source terms in a consistent and stable way, one is also concerned by numerically preserving the equilibrium states of the system (2.3) with source terms: not only steady states at rest $u=0, \partial_{x}(h+b)=\tan \theta, \sigma_{x x}=1=\sigma_{z z}$ here, but also uniform equilibrium $u=U \in \mathbb{R}, g h \tan \theta=k U, \sigma_{x x}=1=\sigma_{z z}$ (well-balancing property, see e.g. [3]). 


\subsection{A difficult well-balancing}

After discretizing the topography in cells as $b_{i}, i \in \mathbb{Z}$, one can consider fully-discrete numerical schemes where source terms are discretized at interfaces through numerical fluxes $F_{l}, F_{r}$ that depend on $\Delta b_{i+1 / 2}=b_{i+1}-b_{i}$

$$
q_{i}^{n+1}=q_{i}^{n}-\frac{\Delta t^{n}}{\Delta x_{i}}\left(F_{l}\left(q_{i}^{n}, q_{i+1}^{n}, \Delta b_{i+1 / 2}, \Delta x_{i+1 / 2}\right)-F_{r}\left(q_{i-1}^{n}, q_{i}^{n}, \Delta b_{i-1 / 2}, \Delta x_{i-1 / 2}\right)\right) .
$$

Such weakly consistent schemes [3] are well-balanced if discrete equilibrium states $q_{i}, i \in \mathbb{Z}$, are preserved:

$$
F_{l}\left(q_{i}, q_{i+1}, \Delta b_{i+1 / 2}, \Delta x_{i+1 / 2}\right)=F_{r}\left(q_{i-1}, q_{i}, \Delta b_{i-1 / 2}, \Delta x_{i-1 / 2}\right) .
$$

When $k=0=\theta$, combining (i) the hydrostatic reconstruction method [1] for the topography source term with (ii) an implicit-in-time discretization of the - linear - dissipative viscoelastic source term (in the second stage of the splitting approach) yields a scheme that is well-balanced as concerns steady states at rest, see [4]. To build a scheme that is well-balanced as concerns the steady states at rest and the new stationary solutions when $k>0, \theta \in\left[0, \frac{\pi}{2}\right)$, one may thus simply want to slightly modify the standard hydrostatic reconstruction method. For instance, one could think of using

$$
q_{i}^{n+1}=\frac{\Delta t^{n} \bar{q}_{i}^{n+1,-}+\lambda\left(q_{i}^{n}-\frac{\Delta t^{n}}{\Delta x_{i}}\left(\mathcal{F}_{l}\left(\left[q_{l}^{\sharp}\right]_{i}^{n},\left[q_{r}^{\sharp}\right]_{i+1}^{n}\right)-\mathcal{F}_{r}\left(\left[q_{l}^{\sharp}\right]_{i-1}^{n},\left[q_{r}^{\sharp}\right]_{i}^{n}\right)+\left(0, \frac{g}{2}\left(\left|\left[h_{l}^{\sharp}\right]_{i}^{n}\right|^{2}-\left|\left[h_{r}^{\sharp}\right]_{i}^{n}\right|^{2}\right), 0,0\right)\right)\right)}{\lambda+\Delta t^{n}} .
$$

where $\bar{q}_{i}^{n+1,-}:=h_{i}^{n+1,-}\left(1, u_{i}^{n+1,-}, 1,1\right)$, where $\left[q_{l / r}^{\sharp}\right]_{i}^{n}$ are the values in cell $i \in \mathbb{Z}$ at time $n=0 \ldots N$ of hydrostatic states $q_{l / r}^{\sharp}=h_{l / r}^{\sharp}\left(1, u, \sigma_{x x}, \sigma_{z z}\right)$, using $h_{l / r}^{\sharp}$, defined with $x_{+} \equiv \max (0, x)$ and $x_{-} \equiv \min (0, x)$ by

$$
\left[h_{l}^{\sharp}\right]_{i}^{n}=\left(h_{i}^{n}-\left(\Delta b_{i+1 / 2}-\left(\tan \theta-\frac{k u_{i}^{n}}{g h_{i}^{n}}\right) \Delta x_{i+1 / 2}\right)_{+}\right)_{+} \quad\left[h_{r}^{\sharp}\right]_{i}^{n}=\left(h_{i}^{n}-\left(\Delta b_{i-1 / 2}-\left(\tan \theta-\frac{k u_{i}^{n}}{g h_{i}^{n}}\right) \Delta x_{i-1 / 2}\right)_{-}\right)_{+} .
$$

The scheme resulting from (3.4) is obviously well-balanced. And it forcefully preserves the invariant domain, since (3.4) is a convex combination in $\mathcal{U}$, even though only a weak version of the free-energy dissipation $\partial_{t}(E+$ $g b h) \leq 0$ is likely to hold (i.e. only for isolated Riemann problems) like in [4] for $k=0=\theta$.

In fact, such a scheme was not a good idea in most of our numerical cases, especially because the outer truncation in (3.5), whose sole purpose is to preserve $h \geq 0$ (but does not preserve energy in turn), often acts too much and generates spurious oscillations. Indeed, hydrostatic reconstruction is a good idea mainly for flow regimes close to hydrostatic equilibrium, i.e. close to steady states at rest. It is not a good idea for cases where the kinetic part of the energy is not negligible, see also e.g. [6]. That is why, although we use a similar time-splitting approach as in [4] with exactly the same first stage, we do not treat the topography source term in the same way in our numerical simulations. Let us see a different way of computing the effect of the source terms during the second stage of our splitting approach.

\subsection{Implicit source terms}

Starting from the intermediate-step cell values $q_{i}^{n+1,-}$, which satisfy a discrete free-energy dissipation $\left(E_{i}^{n+1} \equiv\right.$ $\left.E\left(q_{i}^{n+1,-}\right) \leq E\left(q_{i}^{n}\right) \equiv E_{i}^{n}\right)$, we use a scheme where source terms are discretized semi-implicitly in cells as follows:

$$
\begin{aligned}
h_{i}^{n+1} & =h_{i}^{n+1,-}, \\
\left(1+\Delta t^{n} k_{i}^{n}\right) u_{i}^{n+1} & =u_{i}^{n+1,-}-\Delta t^{n} \tilde{g}\left(\Delta b_{i} / \Delta x_{i}-\tan \theta\right), \\
\left(\lambda+\Delta t^{n}\right) \sigma_{x x, i}^{n+1} & =\lambda \sigma_{x x, i}^{n+1,-}+\Delta t^{n} \\
\left(\lambda+\Delta t^{n}\right) \sigma_{z z, i}^{n+1} & =\lambda \sigma_{z z, i}^{n+1,-}+\Delta t^{n} .
\end{aligned}
$$


In (3.6), we have denoted $k_{i}^{n}$ a function of $q_{i}^{n}$, and cell values $\Delta b_{i}=b_{i+\frac{1}{2}}-b_{i-\frac{1}{2}}$, after discretizing the topography as $b_{i+\frac{1}{2}}, i \in \mathbb{Z}$, at interfaces. (This is as easy and as natural as cell discretizations $b_{i}$ in 1D geometries.)

The resulting scheme is not well-balanced, but it satisfies the fully-discrete free-energy-"dissipation" inequality

$$
\begin{array}{r}
\frac{E_{i}^{n+1}-E_{i}^{n}}{\Delta t^{n}}+\frac{h_{i}^{n+1}\left|u_{i}^{n+1}-u_{i}^{n+1,-}\right|^{2}}{2}+\frac{\mathcal{G}\left(q_{i-1}^{n}, q_{i}^{n}\right)-\mathcal{G}\left(q_{i}^{n}, q_{i+1}^{n}\right)}{\Delta x_{i}}+\tilde{g}\left(b_{i}-x_{i} \tan \theta\right) \frac{h_{i}^{n+1}-h_{i}^{n}}{\Delta t^{n}} \\
+\frac{\tilde{g}}{\Delta x_{i}}\left(h_{i}^{n+1} u_{i}^{n+1}\left(\Delta b_{i}-\Delta x_{i} \tan \theta\right)+\left(b_{i}-x_{i} \tan \theta\right)\left(\mathcal{F}_{l}^{h}\left(q_{i}^{n}, q_{i+1}^{n}\right)-\mathcal{F}_{r}^{h}\left(q_{i-1}^{n}, q_{i}^{n}\right)\right)\right) \\
\leq h_{i}^{n+1}\left(\frac{G}{4} \frac{4-\operatorname{tr} \boldsymbol{\sigma}_{i}^{n+1}-\operatorname{tr}\left[\boldsymbol{\sigma}_{i}^{n+1}\right]^{-1}}{\lambda}-k_{i}^{n}\left|u_{i}^{n+1}\right|^{2}\right)
\end{array}
$$

where $\mathcal{G}$ is exactly the same discrete free-energy flux as in [4].

Of course, the topography source term in (3.7) has no sign, and (3.7) is not actually a dissipative inequality. It is thus not a pointwise stability criterion either. Though, it is consistent with the continuous free-energy inequality satisfied by smooth solutions to (2.3). And the following inequality holds for $\epsilon>0$

$$
\left(1+\Delta t^{n} \min \left(\frac{1}{2 \lambda}, 2\left(\min _{i} k_{i}^{n}-\epsilon\right)\right)\right) \sum_{i \in \mathbb{Z}} E_{i}^{n+1} \leq \sum_{i \in \mathbb{Z}} E_{i}^{n}+\frac{1}{\epsilon} \Delta t^{n} C_{g}
$$

where $C_{g}:=\sum_{i \in \mathbb{Z}}\left|\tilde{g}\left(\Delta b_{i} / \Delta x_{i}-\tan \theta\right)\right|^{2} \geq 0$, assuming no energy flux from infinity. Eq. (3.8) shows that the total free-energy cannot grow infinitely in finite time when $\min _{i} k_{i}^{n}>0$ and $C_{g}<\infty$ (the case of slowly-varying topographies). And we did not observe spurious oscillations with the scheme above in our numerical test cases.

\subsection{Results}

In order to numerically explore the new model, we do not limit to cases where $G$ and $k$ (in fact, Manning coefficient $M$ here) are actually small compared with e.g. $\lambda$, but as usual, we rather let the parameter vary in a physically reasonable range so as to treat illustrative test cases.

We consider first a "dam-break" testcase on a flat plane $\partial_{x} b=0, x \in(-5,5)$. The initial states correspond to two "wet" regions locally at rest and separated by a wall at $x=0$ that is instantaneously broken at $t=0$. (The Newtonian case modelled by inviscid shallow-water equations on a flat plane $\theta=0$ without friction $k=0$ has the well-known Stoker analytical solution.) On the left, we set $x \tan \theta+h^{0}=3$, on the right $x \tan \theta+h^{0}=1$. We show close-shots of states at $T=.4$ simulated on a grid made of 200 points regularly spaced (the boundary conditions at $x= \pm 5$ have not played any role at this final time when assuming e.g. zero-flux).

When $\theta=0$ and $M=0$, the solution is similar to that in [4]. We show in Fig. 1 the influence of each new term independently.

One observes that an inclined plane under the flow tends to push a little bit of fluid toward to the shock front. But this effect is all the more moderate as the fluid is elastic (higher elastic stress relaxation number).

Friction slows down the time evolution as $M$ (thus also $k$ ) increases, as expected from the Newtonian case, and smoothens the sharp fronts at the same time. But that latter diffusive effect seems all the more countered by elastic stress when $G$ increases.

On the contrary, the small relaxation time limit also introduces diffusive effects (insofar as viscous dissipation increases when $\lambda$ decreases) but varying $G$ then hardly influences the result.

In any case, whatever the quality of the model at adequately describing free-surface viscoelastic flows, one could also observe that our numerical scheme is robust, since it has allowed us to explore the influence of the various physical parameters in large ranges so far (the discrete solutions converged with respect to the mesh size in all cases). 

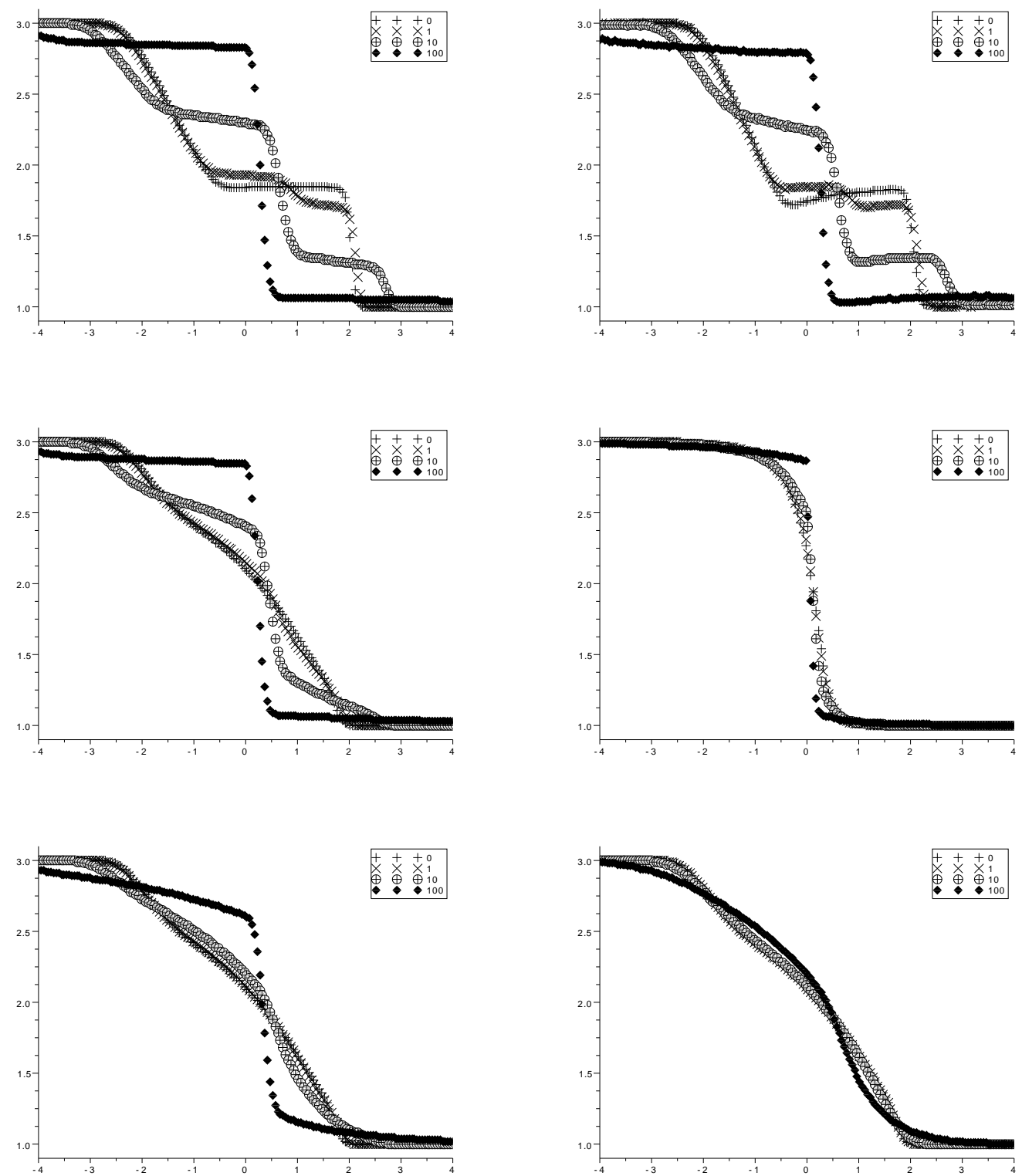

Figure 1. Free surfaces $b+h$ for various elastic stress relaxation numbers $G=0,1,10,100$, as precised in the legends of the figures (hereafter commented, left first then right, within each row from top to bottom) (i) for $\theta=0$ with $M=0$ when relaxation time is large $\lambda=1$ (ii) for $\theta=20^{\circ}$ with $M=0$ when relaxation time is large $\lambda=1$ (iii) for $\theta=0$ with $M=1$ when relaxation time is large $\lambda=1$ (iv) for $\theta=0$ with $M=10$ when relaxation time is large $\lambda=1$ (v) for $\theta=0$ with $M=1$ when relaxation time is small $\lambda=.1$ (vi) for $\theta=0$ with $M=1$ when relaxation time is smaller $\lambda=.01$. 
Second, let us consider a case where the new friction term plays a key role. In particular, a uniform flow with non-zero Froude number $\mathrm{Fr}=u^{0} / \sqrt{g h^{0}}$ is a stationary solution to our new reduced model on a flat rugous inclined plane given non-zero $\theta$ and $M$ if the flow depth satisfies $h^{0}=(g F r M)^{2} / \tan \theta^{3}$, and if it holds $\sigma_{x x}^{0}=1=\sigma_{z z}^{0}$. Now, we observed numerically that our scheme can indeed preserve a stationary uniform flow. But the latter is not exactly the equilibrium state of the continuous model, since the scheme is not well-balanced. Yet, it converges to the good equilibrium as the mesh size is refined, at least for small enough $\mathrm{Fr}$.

We also consider the evolution of an initial small perturbation of a stationary uniform flow on a flat rugous inclined plane $\left(\theta=20^{\circ}, M=.1\right)$ with periodic boundary conditions. A bifurcation of small perturbations toward non-uniform stationary solutions has been identified for Newtonian fluids $(G=0)$ when $F r>2$ [9]. This is often interpreted as an occurence of the roll-waves observed in nature [7]. Now, in the Newtonian case $(G=0)$, we indeed observe numerically the "long-time" convergence (at $T=1$ ) of an initial periodic perturbation of the stationary uniform flow $F r=4$ obtained after multiplication by $1+.01 \sin (K x(2 \pi / 10))$ on $x \in(-5,5)$, toward something similar to a roll-wave, with the number of initial "oscillations" $K=3$ maintained by the limit solution. And the convergence of the "roll-wave" solution as the mesh size is refined (Fig.2) seems to confirm that one may expect other stationary solutions than uniform stationary states, when $F r$ is large enough.
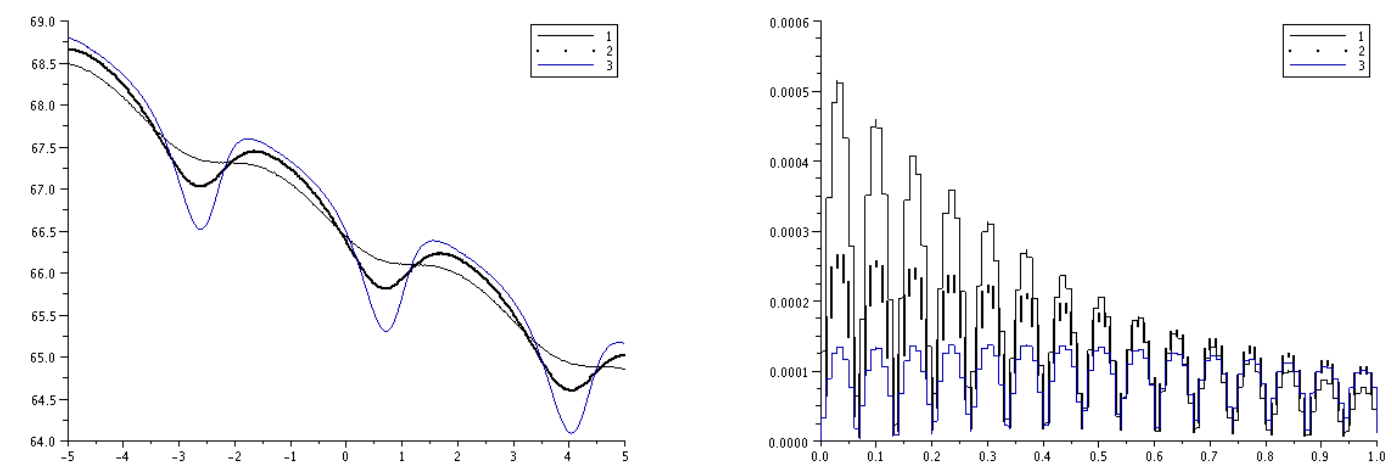

Figure 2. Left: free surfaces $b+h$ of Newtonian thin-layer flows for $F r=4, \theta=20^{\circ}, M=.1$ obtained at $T=1$ when starting from a multiplicative pertubation $1+.01 \sin (K x(2 \pi / 10))$, $K=3$, of the stationary uniform flow, for various refinements $-500,1000$ and 2000 points (1, 2 and 3 resp.) -. Right: time evolution of the relative difference in $\ell^{\infty}$ norm between two successive increments, for the various refinements.

We finally consider similar cases with elastic stress relaxation numbers $G=0,1,10,100$, and a relaxation time $\lambda=1$. The scheme remains robust, and the same "roll-wave" solutions occur. Moreover, viscoelastic effects show up in quite an interesting way. The fluid is pushed differently by gravity along the slope depending whether $G \leq 1$ or $G \geq 10$ (Fig.3).

\section{Conclusion}

The reduced model derived in [4] for shallow viscoelastic free-surface gravity flows has been improved to take into account inclined gravity effects, and friction at a rugous bottom boundary. The new reduced model is one variation of [4] among the many possible ones reviewed in [5]. It is however derived a bit differently here than in [5]. And the absence of bulk dissipation in the initial full model implies that a different scaling of the flow regime is required for consistency of the simplification procedure.

To illustrate numerically the new model, we have followed the FV approach of [4], but an alternative discretization of the source terms (other than the hydrostatic reconstruction of [4]) has been used. The proposed 

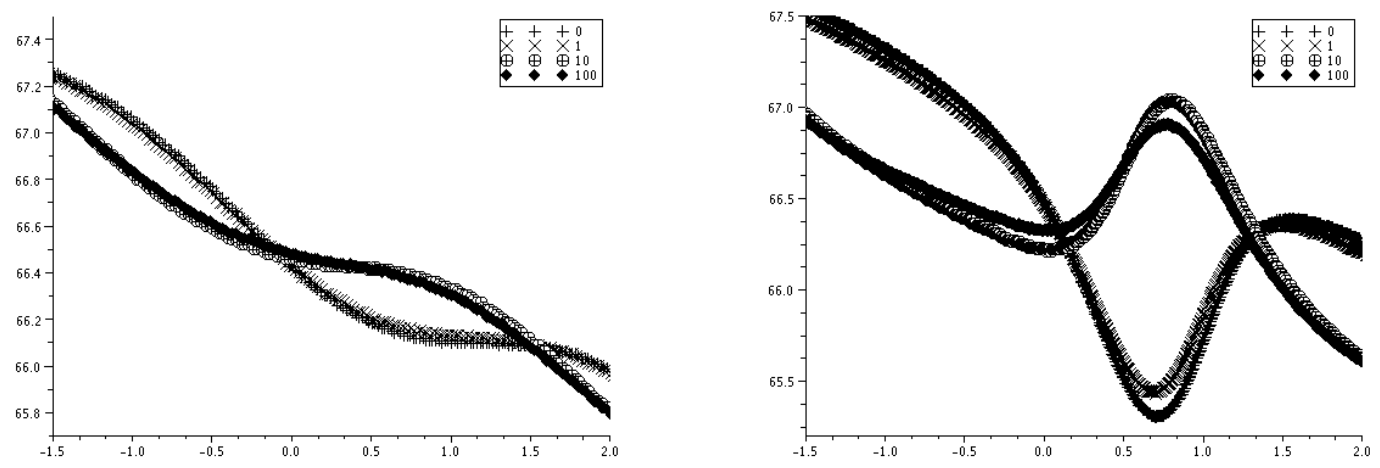

Figure 3. Free surfaces $b+h$ of viscoelastic thin-layer flows for $F r=4, \theta=20^{\circ}, M=.1$ obtained at $T=1$ when starting from a multiplicative pertubation $1+.01 \sin (K x(2 \pi / 10))$, $K=3$, of the stationary uniform flow, for $G=0,1,10,100$. Left: with 500 points - Right: with 2000 .

method is not exactly well-balanced, but it allows one to approximately preserve old and new physicallymeaningful stationary solutions of the new model. The numerical method also proved robust. Additionally, long-time simulations have given indications for stationary solutions of roll-wave type, which we believe to be new in the case of viscoelastic fluids. This should explored in more details in the future.

\section{REFERENCES}

[1] Emmanuel Audusse, François Bouchut, Marie-Odile Bristeau, Rupert Klein, and Benoît Perthame, A fast and stable wellbalanced scheme with hydrostatic reconstruction for shallow water flows, SIAM J. Sci. Comput. 25 (2004), no. 6, 2050-2065. MR MR2086830 (2005f:76069)

[2] François Bouchut, Entropy satisfying flux vector splittings and kinetic bgk models, Numerische Mathematik 94 (2003), 623-672, 10.1007/s00211-002-0426-9.

[3] , Nonlinear stability of finite volume methods for hyperbolic conservation laws and well-balanced schemes for sources, Frontiers in Mathematics, Birkhäuser Verlag, Basel, 2004. MR MR2128209 (2005m:65002)

[4] François Bouchut and Sébastien Boyaval, A new model for shallow viscoelastic fluids, M3AS 23 (2013), no. 08, $1479-1526$.

[5] __ Unified formal reduction for fluid models of free-surface shallow gravity-flows, Tech. report, Jun 2013, HAL \& ArXiV preprints.

[6] Olivier Delestre, Stéphane Cordier, Frédéric Darboux, and François James, A limitation of the hydrostatic reconstruction technique for shallow water equations, Comptes Rendus Mathematique 350 (2012), no. 13-14, $677-681$.

[7] Robert F. Dressler, Mathematical solution of the problem of roll-waves in inclined opel channels, Communications on Pure and Applied Mathematics 2 (1949), no. 2-3, 149-194.

[8] Jean-Frédéric Gerbeau and Benoît Perthame, Derivation of viscous Saint-Venant system for laminar shallow water ; numerical validation, Discrete and continuous dynamical system Series B 1 (2001), no. 1, 89-102.

[9] J. Yu and J. Kevorkian, Nonlinear evolution of small disturbances into roll waves in an inclined open channel, Journal of Fluid Mechanics 243 (1992), 575-594. 